of dried nitrogen was bubbled through the flask before and during the arcing. Around $500 \mathrm{cc}$ of a sol with a concentration of nickel of the order given in the table was produced by about twenty minutes of arcing. The radii of the particles were determined by a rough count with a slit ultramicroscope. Measurements on the hydrosols were made as soon as possible after they were prepared as the value of the intensity of magnetization decreased with time as the nickel oxidized and in five or six days was indistinguishable from that of water.

The extremely small percentage of uncombined nickel determined by these measurements is rather surprising. Hatshek and Thorne $^{3}$ have published analyses of the material deposited on electrodes in a cataphoresis experiment on benzene-carbonyl sols of this type. They treated the material with hydrochloric acid in a nitrometer and measured the total amount of hydrogen evolved. From this they computed the proportion of uncombined nickel, and obtained values in the neighborhood of 80 percent of the total nickel. In no case have magnetic methods of measuring the purity given values over 7 percent.

Hatshek and Thorne report the only other impurity in their sols as a carbonate of nickel. In the above table, while sol $\mathrm{A}$ was prepared by the method used by these authors, sol B had any possible admixture of $\mathrm{CO}_{2}$ removed from the gaseous mixture of $\mathrm{CO}$ and nickel carbonyl by absorption in soda lime just before the nickel carbonyl was dissociated. The percentage of metallic nickel of the sols was improved by this, but it still remained very low.

It is possible to explain this discrepancy otherwise than by contamination of the material used in the present experiments. It is now well recognized that the particles in metal sols obtain their charges by the adsorption of ions. The forces which hold these ions must be chemical in nature, since the adsorption is selective. Now it is quite reasonable to suppose that this weak binding is sufficient to destroy the ferromagnetic properties of nickel, and at the same time is so weak that chemical or mechanical manipulations will break it down.

It would be extremely desirable to obtain further information relative to the validity of this supposition, as it should throw light upon some of the well known, but poorly understood, phenomena of ferromagnetism and ion adsorption.

Carol G. Montgomery

Sloane Physics Laboratory, Yale University, December 12, 1931.

\title{
A Hydrogen Isotope of Mass 2
}

The proton-electron plot of known atomic nuclei shows some rather marked regularities among atoms of lower atomic number. ${ }^{1} \mathrm{Up}$ to $\mathrm{O}^{16}$ a simple step-wise figure appears into which the nuclear species $\mathrm{H}^{2}, \mathrm{H}^{3}$ and $\mathrm{He}^{5}$ could be fitted very nicely. Birge and Menzel ${ }^{2}$ have shown that the discrepancy between the chemical atomic weight of hydrogen and Aston's value by the mass spectrograph could be accounted for by the assumption of a hydrogen isotope of mass 2 present to the extent of 1 part in 4500 parts of hydrogen of mass 1 .

It is possible to calculate with confidence the vapor pressures of the pure substances $\mathrm{H}^{1} \mathrm{H}^{1}, \mathrm{H}^{1} \mathrm{H}^{2}, \mathrm{H}^{1} \mathrm{H}^{3}$, in equilibrium with the pure solid phases. It is only necessary to assume that in the Debye theory of the solid state, $\Theta$ is inversely proportional to the square root of the masses of these molecules and that the rotational and vibrational energies of the molecules do not change in the process of vaporization. These assumptions are in accord with well-established experimental evidence. We find that the vapor pressures for these three molecules in equilibrium with their solids should be in the ratio of $p_{11}: p_{12}: p_{13}=$ $1: 0.37: 0.29$. The theory of the liquid state is not so well understood but it seems reasonable to believe that the differences in vapor pressure of these molecules in equilibrium with their liquids should be rather large and should make possible a rapid concentration of the heavier isotopes, if they exist, in the residue from the simple evaporation of liquid hydrogen near its triple point.

Accordingly two samples of hydrogen were prepared by evaporating large quantities of liquid hydrogen and collecting the gas which evaporated from the last fraction of the last

${ }^{1}$ Urey, J. Am. Chem. Soc. 53, 2872 (1931); Johnston, ibid., 53, 2866 (1931).

${ }^{2}$ Birge and Menzel, Phys. Rev. 37, 1669 (1931). 
cubic centimeter. The first sample was collected from the end portion of six liters of liquid evaporated at atmospheric pressure, and the second sample from four liters evaporated at a pressure only a few millimeters above the triple point. The process of liquefaction has probably no effect in changing the concentration of the isotopes since no appreciable change was observed in the sample evaporated at atmospheric pressure.

These samples were investigated for the atomic spectra of $\mathrm{H}^{2}$ and $\mathrm{H}^{3}$ in a hydrogen discharge tube run in Wood's so-called "black stage" by using the second order of a 21 foot grating with a dispersion of $1.31 \mathrm{~A}$ per $\mathrm{mm}$. With the sample evaporated at the boiling point no concentration so high as had been estimated was detected. We then increased the exposures so that the ratio of the time of exposure to the minimum required to get the $\mathrm{H}^{1}$ lines on our plates was about $4500: 1$. Under these conditions we found in this sample as well as in ordinary hydrogen faint lines at the calculated positions for the lines of $\mathrm{H}^{2}$ accompanying $H_{\beta}, H_{\gamma}, H_{\delta}$. These lines do not agree in wave-length with any molecular lines reported in the literature. ${ }^{3}$ However they were so weak that it was difficult to be sure that they were not ghosts of the strongly overexposed atomic lines.

The sample of hydrogen evaporated near the triple point shows these lines greatly enhanced, relative to the lines of $\mathrm{H}^{1}$, over both those of ordinary hydrogen and of the first sample. The relative intensities can be judged by the number and intensity of the symmetrical ghosts on the plates. The wave-lengths of the $\mathrm{H}^{2}$ lines appearing on these plates could be easily measured within about $0.02 \mathrm{~A}$. The following table gives the mean of the observed displacements of these lines from those of $\mathrm{H}^{1}$ and the calculated displacements: ghosts of the respective $\mathrm{H}^{1}$ lines appear nearly constant for any one sample of hydrogen, they are not ghosts for their intensities relative to the known ghosts are not the same in the case of ordinary hydrogen and of the 1st sample as they are in the case of the second sample. They are not molecular lines for they do not appear on a plate taken with the discharge tube in the "white stage" with the molecular spectrum enhanced $\left(\mathrm{H}^{2} \gamma\right.$ was found as a slight irregularity on a microphotometer curve of this plate). Finally the $\mathrm{H}^{2}{ }_{\alpha}$ line is resolved into a doublet with a separation of about $0.16 \mathrm{~A}$ in agreement with the observed separation of the $\mathrm{H}^{1}{ }_{\alpha}$ line.

The relative abundance in ordinary hydrogen, judging from relative minimum exposure time is about 1:4000, or less, in agreement with Birge and Menzel's estimate. A similar estimate of the abundance in the second sample indicated a concentration of about 1 in 800. Thus an appreciable fractionation has been secured as expected from theory. ${ }^{4}$

No evidence for $\mathrm{H}^{3}$ has been secured, but its lines would fall on regions of our plates where the halation is bad.

The distillation was carried out at the $\mathrm{Bu}$ reau of Standards by one of us (F.G.B.), who is continuing the fractionation to secure more highly concentrated samples. The spectroscopic work was done at Columbia University by the other two (H.C.U. and G.M.M.) who are working on the molecular spectrum.

$$
\begin{aligned}
& \text { Harold C. Urey } \\
& \text { F. G. BrickWedde } \\
& \text { G. M. Murphy }
\end{aligned}
$$

Columbia University,

New York, N. Y.

Bureau of Standards,

Washington, D. C.

December 5, 1931.

\begin{tabular}{l|c|c|c|c}
\hline \multicolumn{1}{c|}{ Line } & $H_{\alpha}$ & $H_{\beta}$ & $H_{\gamma}$ & $H_{\delta}$ \\
\hline$\Delta \lambda$ calc. & 1.793 & 1.326 & 1.185 & 1.119 \\
$\Delta \lambda$ obs. & - & 1.346 & 1.206 & 1.145 \\
$\quad$ Ordinary hydrogen & $\overline{-}$ & 1.330 & 1.199 & 1.103 \\
1st sample & 1.820 & 1.315 & 1.176 & - \\
2nd sample &
\end{tabular}

The $\mathrm{H}^{2}$ lines are broad, as is to be expected for close unresolved doublets, but they are not as broad and diffuse as the $\mathrm{H}^{1}$ lines probably due to the smaller Döppler broadening. Although their intensities relative to the
${ }^{3}$ Gale, Monk and Lee, Astrophys. J. 57, 89 (1928); Finkelnburg, Z. Physik 52, 57 (1928); Connelly, Proc. Phys. Soc. 42, 28 (1929).

${ }^{4}$ Keesom and van Dijk, Proc. Acad. Sci. Amsterdam 34, 52 (1931). 